\title{
Las chalconas y su uso como precursores en la síntesis de compuestos heterocíclicos nitrogenados
}

\author{
Braulio Insuasty-Obando \\ Departamento de Química, Facultad de Ciencias Naturales y Exactas, \\ Universidad del Valle, Cali, Colombia
}

Artículo de posesión para el ingreso como miembro correspondiente a la

Academia Colombiana de Ciencias Exactas, Físicas y Naturales el 2 de diciembre de 2015

\begin{abstract}
Resumen
La síntesis de nuevos compuestos con posible actividad biológica es un gran reto de la investigación en química orgánica y medicinal y es de gran impacto debido a las posibilidades de desarrollo de nuevas alternativas terapéuticas para el tratamiento de diversos tipos de enfermedades. En este documento se presenta un resumen del trabajo investigativo realizado por el profesor Braulio Insuasty Obando y sus colaboradores en los últimos años en la línea de investigación de síntesis de compuestos heterocíclicos nitrogenados de 5, 6 y 7 miembros (pirazolinas, piridinas y diazepinas) con posible actividad biológica a partir de 1,3-difenil-2-propen-1-onas (chalconas) y sus análogos. La preparación y caracterización de los nuevos compuestos se realizó en el Grupo de Investigación de Compuestos Heterocíclicos de la Universidad del Valle. Para llevar a cabo las reacciones de síntesis, además de las fuentes de energía convencionales (calentamiento a reflujo) se utilizaron fuentes no convencionales como la radiación de microondas, ultrasonido. Algunas reacciones se llevaron a cabo utilizando metodologías amigables con el medio ambiente como: reacciones libres de disolvente y reacciones multicomponentes. El uso de estas metodologías sintéticas nos permite realizar reacciones más limpias, en menor tiempo y con mejores rendimientos. Los compuestos sintetizados se caracterizaron usando técnicas espectroscópicas como FT-IR, RMN-1D y 2D, espectrometría de masas, análisis elemental y difracción de rayos X en algunos casos. (C) Acad. Colomb. Cienc. Ex. Fis. Nat. 2016.
\end{abstract}

Palabras clave: orto-diaminas, chalconas, pirazolinas, piridinas, diazepinas, actividad biológica.

Chalcones and their use as precursors in the synthesis of nitrogen-containing heterocyclic compounds

\begin{abstract}
The synthesis of new compounds with potential biological activity is a major challenge for research in organic and medicinal and is of great impact due to the potential development of new therapies for the treatment of various types of diseases. This document is a summary of the research conducted by Professor Braulio Insuasty Obando and his group in recent years in the topic of synthesis of 5, 6 and 7 members nitrogen-containing heterocyclic compounds (pyrazolines, pyridines and diazepines) with potential biological activity starting from 1,3-diphenyl2-propen-1-ones (chalcones) and its analogues. The preparation and characterization of the new compounds was conducted in the Grupo de Investigación de Compuestos Heterocíclicos of the Universidad del Valle. To carry out the synthesis reactions, besides conventional energy sources (refluxing) unconventional sources were used such as microwave irradiation and ultrasound. Some reactions were carried out using environmental friendly methods as solvent-free reactions and multicomponent reactions. Use of these synthetic methodologies allows making cleaner reactions, in less time and with better yields. The synthesized compounds were characterized using spectroscopic techniques such as FT-IR, 1D and 2D NMR, mass spectrometry, elemental analysis and X-ray diffraction in some cases. (c) Acad. Colomb. Cienc. Ex. Fis. Nat. 2016.
\end{abstract}

Key words: Ortho-diamines, chalcones, pyrazolines, pyridines, diazepines, biological activity.

\section{Introducción}

Las chalconas 1 son cetonas aromáticas $\alpha, \beta$-insaturadas de origen natural o sintético (Figura 1), denominadas también como bencilidenacetofenonas, estiril-fenil-cetonas, benzalacetofenonas, diarilpropenonas o 1,3-difenil-2-propen1-onas según la IUPAC.

Las orto-hidroxichalconas 2 ( Figura 1S, http://www.raccefyn. co/index.php/raccefyn/article/downloadSuppFile/309/1471), 
son precursores para la síntesis de flavonas 3, pigmentos naturales ampliamente difundidos. (Awad, El-Neweihy y Selim, 1960).

Algunas chalconas presentan actividad antimalárica, antileishmaniasis, antituberculosa, antifúngica, antibacterial y antitumoral. (Parvesh, Amit y Vipan, 2014).

Las chalconas son compuestos de una gran reactividad debido a la presencia de dos centros electrofílicos, los cuales pueden actuar como una sola unidad o de manera independiente lo que permite para estos sistemas el ataque de nucleófilos y la posibilidad de sufrir una adición 1,2(adición directa) (A) o 1,4- (adición conjugada) (B), ( Figura 2S, http://www.raccefyn.co/index.php/raccefyn/article/ downloadSuppFile/309/1472).

El comportamiento químico de las chalconas es similar al de los compuestos 1,3-dicarbonílicos, $\mathrm{RCOCH}_{2} \mathrm{COR}$ ', los cuales se caracterizan por presentar dos centros electrofílicos. La diferencia entre estos últimos y las chalconas se considera en la diferencia de reactividad de los dos centros. En el caso de los compuestos 1,3-dicarbonílicos debido a la poca diferencia de sus centros reactivos, en la reacción de ciclocondensación con dinucleófilos, se pueden obtener mezclas de productos, en muchos casos difíciles de separar por su similitud en propiedades. Una de las ventajas de utilizar las chalconas en este tipo de reacciones es la formación en la mayoría de casos de un solo producto y otra ventaja adicional es que su uso nos permite obtener sistemas parcialmente hidrogenados a diferencia del caso de los compuestos 1,3-dicarbonílicos.

La propiedad anteriormente mencionada, es muy útil para la construcción de sistemas heterocíclicos de 5, 6 y 7 miembros, por ejemplo, pirazolinas, isoxazoles, piridinas, pirimidinas y diazepinas (Figura 2).

Estos compuestos heterocíclicos presentan un gran espectro de actividad biológica como: antibacterial, antifúngica, antitumoral, antimalárica, antiviral, antiinflamatoria, antitubercular, analgésica, antioxidante y antipsicótica. (Mohamed, Abdelrahman, y Ahmad, 2012) (Anand y Singh, 2014) (Wenglowsky, 2013) (Ramajayam, Girdhar, y Yadav, 2007).

\section{Síntesis de chalconas y análogos}

Aunque existen varios métodos para la síntesis de chalconas, tal vez el método más simple y versátil es mediante la condensación de Claisen-Schmidt entre derivados de la acetofenona y benzaldehídos, bien puede ser en medio básico o ácido. (Kostanecki y Tambor, 1899). Este método permite introducir una gran variedad de sustituyentes en los anillos

\section{Correspondencia:}

Braulio Insuasty-Obando, braulio.insuasty@correounivalle.edu.co Recibido: 12 de noviembre de 2015

Aceptado: 23 de mayo de 2016

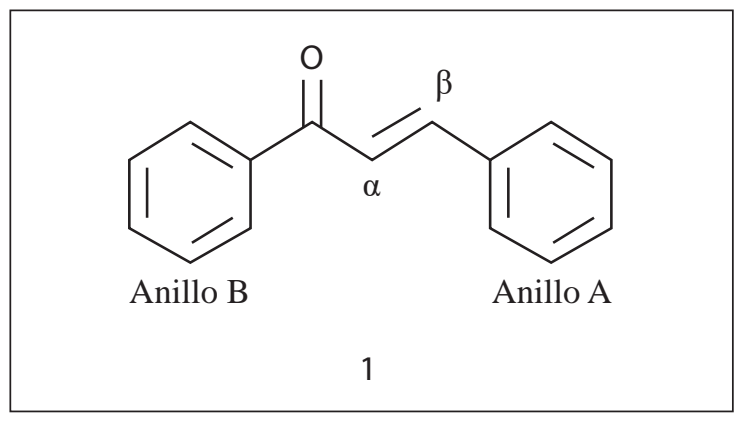

Figura 1. Estructura general de las chalconas.

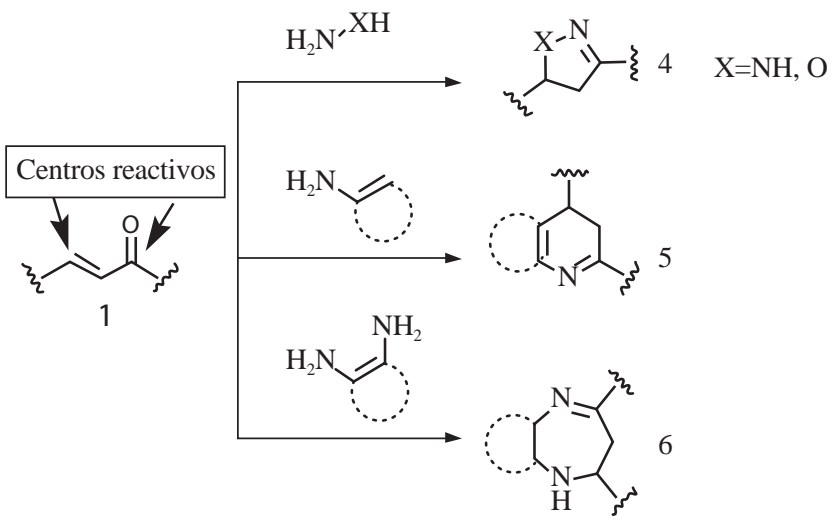

Figura 2. Obtención de sistemas heterocíclicos de 5 (4), 6 (5) y 7 miembros (6) a partir de sistemas carbonílicos $\alpha, \beta$-insaturados.

de las chalconas y conlleva a la formación del producto de configuración E (Figura 3S, http://www.raccefyn.co/index. php/raccefyn/article/downloadSuppFile/309/1473).

Insuasty et al. (2015) reportaron la síntesis de una nueva serie de chalconas 9a-f a partir del aldehído derivado de la cafeína 7 y acetofenonas sustituidas 8a-f. Los compuestos $\mathbf{9 b}$ y 9 e mostraron remarcable actividad antiprotozoaria contra Leishmania panamensis con inhibición del crecimiento de $88.3 \pm 1.5$ y $82.6 \pm 2.2 \%$ respectivamente, a $20 \mu \mathrm{g} / \mathrm{mL}$. Los ensayos in vitro contra Trypanozoma cruzi mostraron que la chalcona 9f fue considerada especialmente activa con una inhibición del crecimiento de $9.7 \pm 1.5 \%$, para una muy baja concentración de 1,0 $\mu \mathrm{g} / \mathrm{mL}$ (Figura 3).

Con el objetivo de obtener los compuestos carbonílicos $\alpha, \beta-$ insaturados (chalconas 13/14) como materiales de partida para la síntesis de 2-pirazolinas, se llevó a cabo una condensación de Claisen-Schmidt entre las cetonas 10/11 con diferentes aldehídos aromáticos 12, lo cual condujo a la obtención de las chalconas 13/14 con buenos rendimientos ( Figura 4S, http://www.raccefyn.co/index.php/raccefyn/article/download SuppFile/309/1474). (Insuasty, et al., 2013a).

Nuevas (E)-1-aril-3-(3-aril-1-fenil-1H-pirazol-4-il)-2-propen1-onas 17/18 fueron sintetizadas por medio de una reacción de Claisen-Schmidt entre 3-aril-1-fenilpirazol- 


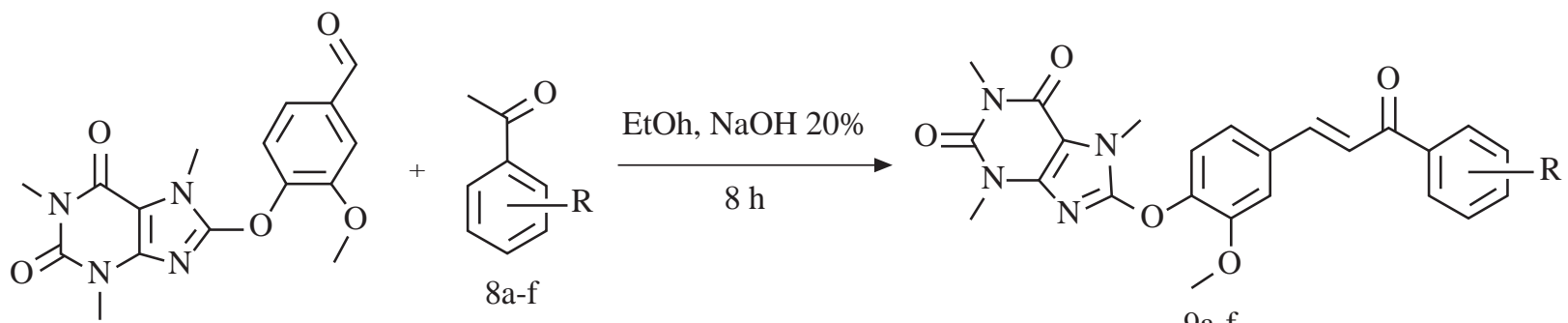

7

$\mathrm{R}:$
a. $4-\mathrm{H}$
b. 4-CI
c. $4-\mathrm{Br}$
d. $4-\mathrm{CH}_{3}$
e. $4-\mathrm{OCH}_{3}$
f. 3,4,5- $\left(\mathrm{OCH}_{3}\right)_{3}$

Figura 3. Síntesis de una nueva serie de chalconas 9a-f a partir del aldehído derivado de la cafeína 7 y acetofenonas sustituidas 8a-f.

4-carboxaldehídos 15/16 con diversos derivados de acetofenona 8a-h (Figura 5S, http://www.raccefyn.co/index.php/ raccefyn/article/downloadSuppFile/309/1475). El compuesto 17c mostró una remarcable actividad contra las líneas celulares de leucemia (K-562 y SR), cáncer renal (UO-31) y cáncer de pulmón de células no pequeñas (HOP-92), con los valores más importantes de $\mathrm{GI}_{50}\left(\mathrm{GI}_{50}\right.$ es la concentración en la cual se logra una inhibición del $50 \%$ de la proliferación de células tumorales) en un rango entre 0.04 a $11.4 \mu \mathrm{M}$, para los ensayos in vitro. (Insuasty, et al., 2010a).

Los derivados de tipo bis-chalconas 21 fueron obtenidos por medio de una reacción de Claisen-Schmidt tratando las dicetonas 19/20 con aldehídos aromáticos 12 en etanol con cantidades catalíticas de hidróxido de sodio. Todas las bis-chalconas fueron obtenidas con buenos rendimientos (Figura 6S, http://www.raccefyn.co/index.php/raccefyn/ article/downloadSuppFile/309/1476). (Insuasty, et al., 2008a).

De modo similar, se logró la obtención de metilen-bischalconas 23a-e partiendo de acetofenonas sustituidas 8 y 5,5'-metilen-bis-salicilaldehído 22 (Figura 7S, http://www. raccefyn.co/index.php/raccefyn/article/downloadSuppFile/ 309/1477). (Insuasty, et al., 2011).

Nuevos derivados hetaril- y alquilidenrodaninas 26a-d, 26e, y 27a-d, compuestos carbonílicos $\alpha, \beta$-insaturados, fueron preparados a partir de aldehídos heterocíclicos 24ad o acetaldehído 24e (Figura 8S, http://www.raccefyn.co/ index.php/raccefyn/article/downloadSuppFile/309/1478). El tratamiento de los derivados 26a-d y 26e con piperidina o morfolina en THF a reflujo, produjo (Z)-5-(hetarilmetiliden)2-(piperidin-1-il)tiazol-4-(5H)-onas y 2-morfolinotiazol-4(5H)-onas 28a-d, 29a-d, y ( $\mathrm{Z}$ )-5-etiliden-2-morfolinotiazol4-(5H)-ona (28e), respectivamente, con buenos rendimientos. Varios de esos compuestos fueron evaluados en 60 líneas celulares tumorales humanas diferentes. El compuesto 26c mostró alta actividad antitumoral contra HOP-92 (Cáncer de pulmón de células no pequeñas), la cual fue la línea más sensible, con $\mathrm{GI}_{50}=0,62 \mu \mathrm{M}$ y $\mathrm{LC}_{50}>100 \mu \mathrm{M}\left(\mathrm{LC}_{50}\right.$ es la concentración en la cual ocurre la muerte del 50\% de células normales) de los ensayos in vitro. La actividad antifúngica in vitro de los compuestos fue determinada usando 10 cepas fúngicas (Candida albicans; Saccharomyces cerevisiae; Cryptococcus neoformans; Aspergillus niger; Aspergillus fumigatus; Aspergillus flavus; Microsporum gypseum; Trichophyton rubrum; Trychophyton mentagrophyte). El compuesto 26e mostró actividad contra todas las cepas probadas, pero mostró alta actividad contra la levadura de cerveza Saccharomyces cerevisiae (MIC (concentración mínima inhibitoria) 3,9 $\mu \mathrm{g} / \mathrm{mL}$ ). (Insuasty, et al., 2013b).

La síntesis asistida por microondas de nuevas (Z)-5arilidenrodaninas 30a-i (Figura 9S, http://www.raccefyn.co/ index.php/raccefyn/article/downloadSuppFile/309/1479), bajo condiciones libres de disolvente se llevó a cabo y su actividad antifúngica in vitro fue evaluada siguiendo el estándar CLSI (anteriormente NCCLS) contra un panel de hongos estandarizado y otro patogénico clínicamente oportunista. Un análisis de la relación estructura-actividad (SAR) junto con estudios computacionales mostraron que los compuestos más activos (rodaninas sustituidas con

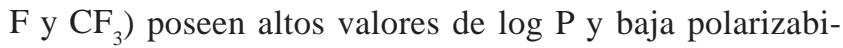
lidad. Ensayos basados en el mecanismo sugieren que los compuestos activos no se unirían al ergosterol ni producirían un daño a la membrana fúngica. (Sortino et al., 2007).

\section{Síntesis de pirazolinas a partir de chalconas}

Los compuestos derivados del anillo de pirazol, parcialmente saturados con un doble enlace en el anillo se conocen como pirazolinas, y existen tres formas diferentes, dependiendo de la posición del doble enlace ( Figura 10S, http://www.raccefyn.co/index.php/raccefyn/article/download SuppFile/309/1480).

Para la síntesis de las 2-pirazolinas, se han reportado varios métodos en la literatura. Sin embargo, la condensación de chalconas sustituidas con derivados de hidrazina es uno de los métodos más populares para la preparación de estos compuestos (Figura 4). 
El tratamiento de los compuestos 9a-f con hidrato de hidrazina condujo a las pirazolinas 33a-f y su posterior reacción con anhídrido acético o ácido fórmico produjo las correspondientes pirazolinas $N$-sustituidas 34a-f y 35a-f respectivamente. En el estudio de actividad in vitro contra Plasmodium falciparum, la pirazolina 33a mostró un porcentaje destacable de inhibición de crecimiento de 85.2 $\pm 5.4 \%$. Los compuestos 34c y $34 \mathrm{f}$ mostraron remarcable actividad contra Leishmania panamensis con inhibición del crecimiento de $82.8 \pm 1.7$ y $87.6 \pm 0.5 \%$ respectivamente, a $20 \mu \mathrm{g} / \mathrm{mL}$. Los ensayos in vitro contra Trypanozoma cruzi para la pirazolina 33d mostraron una inhibición del crecimiento de $61.9 \pm 7.8 \%$ a $20 \mu \mathrm{g} / \mathrm{mL}$ (Figura 5). (Insuasty, et al., 2015).

Una nueva serie de $N H$-pirazolinas 37 ( Figura 11S, http:// www.raccefyn.co/index.php/raccefyn/article/download SuppFile/309/1481), fue preparada mediante la reacción de ciclocondensación de las nuevas [(7-cloroquinolin-4-il)oxi] chalconas 36 con hidrato de hidrazina. El tratamiento de las pirazolinas 37 con anhídrido acético o ácido fórmico condujo a los derivados $N$-acetil- o $N$-formilpirazolinas $\mathbf{3 8 / 3 9}$, respectivamente. Los nuevos derivados 2-pirazolínicos 3739 fueron evaluados por el U.S. National Cancer Institute (NCI). Los compuestos 38b,d,f y 39c,f mostraron interesante actividad antitumoral contra 58 líneas celulares tumorales, con los valores de $\mathrm{GI}_{50}$ más importantes de ensayos in vitro en un rango entre 0.48 a $1.66 \mu \mathrm{M}$. (Montoya, et al., 2014).

El tratamiento de los compuestos 40a-f con hidrato de hidrazina empleando condiciones suaves de reacción condujo a la formación de 4,5-dihidro-1H-pirazoles 41a-f ( Figura 12S, http://www.raccefyn.co/index.php/raccefyn/article/download SuppFile/309/1482). Luego, el tratamiento con anhídrido acético o ácido fórmico produjo los 4,5-dihidro- $1 H$-pirazoles 42a-f y 43a-f esperados. La actividad antifúngica de cada serie de compuestos sintetizados fue determinada contra los hongos clínicamente importantes Candida albicans y Cryptococcus neoformans. Adicionalmente, los compuestos más activos 42e y $42 \mathrm{f}$ fueron probados en combinación con los agentes antifúngicos comerciales: fluconazol, itraconazol y amfotericina B. El compuesto 42e mostró un efecto

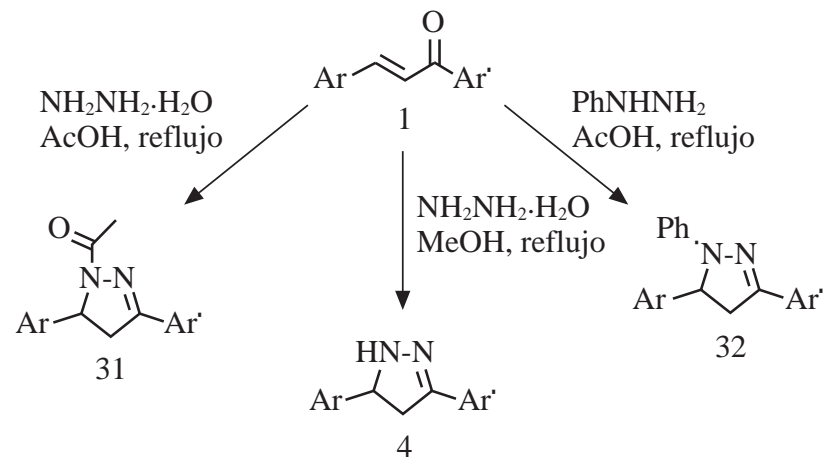

Figura 4. Condensación de las chalconas con hidrazinas para la preparación de 2-pirazolinas. sinérgico con fluconazol contra C. albicans mientras que $\mathbf{4 2 f}$ mostró actividad sinérgica con todos los fármacos antifúngicas probadas contra la misma cepa. (Ramírez, et al., 2014).

Nuevas series de $N$-acetil- 44 y $N$-formil-pirazolinas $\mathbf{4 5 / 4 6}$ (Figura 13S, http://www.raccefyn.co/index.php/raccefyn/ article/downloadSuppFile/309/1483) fueron sintetizadas por reacción de ciclocondensación entre [(7-cloroquinolin4-il)amino]chalconas 13/14 con hidrato de hidrazina en ácido acético y con hidrato de hidrazina en ácido fórmico respectivamente. Los compuestos fueron evaluados in vitro como agentes antitumorales y antimaláricos. Los compuestos 45b y 46b-e mostraron remarcable actividad antitumoral contra líneas celulares tumorales, con los valores más importantes de $\mathrm{GI}_{50}$ en un rango entre 0.13 a $0.99 \mu \mathrm{M}$. La mejor respuesta antimalárica fue observada para el compuesto 45a con un porcentaje de inhibición de 50.8\% para Plasmodium falciparum, una capacidad hemolítica de 3.2\% y un $\mathrm{IC}_{50}$ de $14.1 \mu \mathrm{g} / \mathrm{mL}$. (Insuasty, et al., 2013a).

Se sintetizaron tres series de nuevas 2-pirazolinas 1,3,5-trisustituidas conteniendo el residuo de benzodioxol como potencial agente antitumoral (Figura 14S, http:// www.raccefyn.co/index.php/raccefyn/article/download SuppFile/309/1484). La actividad antitumoral in vitro de los compuestos obtenidos fue determinada en el National Cancer Institute (NCI). La 5-(benzo[d][1,3]dioxol-5-il)-3(4-metoxifenil)-4,5-dihidro-1 $H$-pirazol-1-carbotioamida (54a) es el más prominente de los compuestos debido a su remarcable actividad hacia las líneas celulares de leucemia (RPMI-8226), cáncer renal (UO-31) y cáncer de próstata (DU-145) con valores de $\mathrm{GI}_{50}$ de 1.88, 1.91 y $1.94 \mu \mathrm{M}$, respectivamente. (Insuasty, et al., 2012a).

La reacción de ciclocondensación asistida por microondas de las chalconas 17/18 con hidrazina produjo los nuevos 3-aril-4-(3-aril-4,5-dihidro- $1 \mathrm{H}$-pirazol-5-il)-1-fenil- $1 \mathrm{H}$ pirazoles racémicos 55 o sus $N$-acetil derivados 56 y 57 cuando las reacciones se llevaron a cabo en DMF o ácido acético, respectivamente ( Figura 15S, http://www.raccefyn. co/index.php/raccefyn/article/downloadSuppFile/309/1485). El compuesto $\mathbf{5 7 g}$ mostró una remarcable actividad contra las líneas celulares de leucemia (K-562 y SR), cáncer renal (UO-31) y cáncer de pulmón de células no pequeñas (HOP92), con los valores más importantes de $\mathrm{GI}_{50}$ en un rango entre 0.04 a $11.4 \mu \mathrm{M}$, para los ensayos in vitro. (Insuasty, et al., 2010a).

Nuevos derivados bis-3,5-difenilpirazolínicos 58a-f (Figura 16S, http://www.raccefyn.co/index.php/raccefyn/article/ downloadSuppFile/309/1486), han sido sintetizados de acuerdo a las metodologías de Elway y Sangwan. La mayoría de ellos fueron obtenidos con buenos rendimientos, a partir de la reacción entre bis-chalconas enlazadas por un grupo alquilo 21a-f e hidrazina en ácido acético. La actividad de algunas bis-pirazolinas fue evaluada contra la bacteria Helicobacter pylori. (Insuasty, et al., 2008a). 
<smiles>[R]c1cccc(C2=NNC(c3ccc(Oc4nc5c(c(=O)n(C)c(=O)n5C)n4C)c(OC)c3)C2)c1</smiles>

9a-f

33a-f<smiles>[R]c1cccc(C2=NN(C(C)=O)C(c3ccc(Oc4nc5c(c(=O)n(C)c(=O)n5C)n4C)c(OC)c3)C2)c1</smiles>

$\mathrm{R}:$
a. $4-\mathrm{H}$
d. $4-\mathrm{CH}_{3}$
b. 4-CI
e. $4-\mathrm{OCH}_{3}$
c. $4-\mathrm{Br} \quad$ f. $3,4,5-\left(\mathrm{OCH}_{3}\right)_{3}$

Figura 5. Síntesis de las pirazolinas 33a-f y su posterior reacción con anhídrido acético o ácido fórmico para la obtención de las correspondientes pirazolinas $N$-sustituidas 34a-f y 35a-f.

Dos nuevas series de 4,4'-metilenbis[2-(3-aril-4,5-dihidro- $1 \mathrm{H}$ pirazol-5-il)fenoles] 59 y metilenbis-2-(3-aril-4,5-dihidro$1 H$-pirazol-5-il)-4,1-fenilen diacetatos 60 han sido eficientemente preparados empezando por el salicilaldehído comercialmente disponible y dónde el paso clave corresponde a la reacción asistida por microondas de intermediarios 3,3'-[metilenbis(6-hidroxi-3,1-fenilen)]bis(1-arilprop-2-en1-onas) 23 con hidrato de hidrazina o ácido acético/hidrato de hidrazina respectivamente. Tres compuestos no acetilados tipo 59 presentaron interesante actividad antitumoral contra un amplio rango de líneas celulares tumorales (Figura 17S, http://www.raccefyn.co/index.php/raccefyn/article/download SuppFile/309/1487). (Insuasty, et al., 2011).

\section{Síntesis de piridinas fusionadas a partir de chalconas}

De modo similar, se pueden formar anillos de seis miembros con un átomo de nitrógeno a partir de chalconas. La reacción de ciclocondensación de chalconas con 1,3-binucleófilos, por ejemplo monoaminas de la serie heterocíclica, se presenta como un método simple y versátil para fusionar el anillo piridínico a un heterociclo. El uso de las chalconas en estas reacciones permite obtener los productos parcialmente hidrogenados, o sea derivados dihidropiridínicos fusionados de gran importancia por su actividad biológica (Figura 18S, http://www.raccefyn.co/index.php/raccefyn/article/download SuppFile/309/1488).

Por ejemplo, la condensación de aminopirazoles $\mathrm{NH}$ sustituidos $\mathbf{6 1}$ con chalconas $\mathbf{1}$ conlleva a la formación de dihidropirazolo[3,4-b]piridinas 63. En el caso de aminopirazoles $\mathrm{NH}$ - no sustituidos 62, la introducción de un tercer centro nucleofílico conlleva bien sea a la formación de dihidropirazolo[3,4-b]piridinas 63 o dihidropirazolo [1,5-a]pirimidinas 64, ambas familias de compuestos con importante actividad biológica. Es importante resaltar nuevamente que una de las ventajas de usar las chalconas en lugar de compuestos 1,3-dicarbonílicos, además de la alta regioselectividad que muestran estas reacciones de ciclocondensación con participación de chalconas, se centra en el hecho de poder obtener los dihidroderivados, 
los cuales muestran una marcada estabilidad y se pueden aromatizar mediante su tratamiento con agentes oxidantes o a través de una halogenación-deshidrohalogenación con $N$-bromosuccinimida (Figura 19S, http://www.raccefyn.co/ index.php/raccefyn/article/downloadSuppFile/309/1489). (Quiroga, et al., 1994).

Las 6,7,8,9-tetrahidropirimido[4,5-b][1,6]naftiridin-4(3H, $5 \mathrm{H}, 10 \mathrm{H}$ )-onas 70a-g, 71a-g y sus formas oxidadas 72a-g, 73a-g fueron obtenidas de la reacción libre de catalizador entre 6-amino-2-metiltiopirimidin-4(3H)-ona 69 y $(E)-3,5-$ bis(benciliden)-1-alquil-4-piperidonas 67a-g, 68a-g bajo radiación de microondas y su posterior oxidación con p-cloroanilo. Dieciocho de los nuevos compuestos fueron evaluados en el National Cancer Institute de Estados Unidos, donde el compuesto $\mathbf{7 0 g}$ presentó una remarcable actividad contra 57 líneas celulares tumorales, con los valores más importantes de $\mathrm{GI}_{50}$ entre 1.48 a $9.92 \mu \mathrm{M}$ en ensayos in vitro (Figura 6). (Insuasty, et al., 2013c).

\section{Síntesis de diazepinas fusionadas a partir de chalconas}

Las diazepinas, son compuestos heterocíclicos que contienen en su estructura un anillo de siete miembros con dos átomos de nitrógeno en posiciones [1,2], [1,3] y [1,4] (Figura 20S, http://www.raccefyn.co/index.php/raccefyn/ article/downloadSuppFile/309/1490).

Debido a que este tipo de compuestos se puede obtener por diferentes rutas y el espectro de actividad biológica es muy amplio, se considera el esqueleto diazepínico como base estructural privilegiada y líder para la obtención de nuevas moléculas que exhiban actividad biológica. Muestra de ello, son algunos compuestos de tipo diazepínico como el diazepam y el midazolam que son empleados comercialmente para el tratamiento de la ansiedad y desordenes relacionados con el sistema nervioso central (SNC).

Las chalconas reaccionan con 1,4-dinucleófilos, como orto-diaminas, conllevando a la formación del sistema diazepínico. Es así como la orto-fenilendiamina (Figura 21S, http://www.raccefyn.co/index.php/raccefyn/article/download SuppFile/309/1491), reacciona con chalconas para formar benzodiazepinas. (Insuasty, Abonia y Quiroga, 1992). La reacción transcurre a través de la formación del aducto tipo Michael y posterior condensación.

En el caso de orto-diaminas que contienen dos grupos amino no equivalentes es posible la formación de mezclas de regioisómeros (Figura 22S, http://www.raccefyn.co/ index.php/raccefyn/article/downloadSuppFile/309/1492), la posibilidad de formación de un solo regioisómero depende de la diferencia de nucleofilicidad entre los dos grupos amino. (Vianna-Rodrigues, et al., 1994).

La condensación de chalconas con orto-diaminas de la serie heterocíclica se presenta como un método efectivo para la obtención de dihidrodiazepinas fusionadas. De esta manera se puede anelar el sistema diazepínico a otro heterociclo (Figura 23S, http://www.raccefyn.co/index.php/raccefyn/ article/downloadSuppFile/309/1493).

La ciclocondensación regioselectiva de las chalconas 9a-f con el 4,5-diaminopirazol 79 produjo los derivados diazepínicos 80a-f. En el estudio de actividad in vitro contra Plasmodium falciparum, las diazepinas 80a-f mostraron remarcables porcentajes de inhibición de crecimiento en el rango de 80.3 \pm 13.5 a $94.2 \pm 0.2 \%$ cuando fueron probadas a $20 \mu \mathrm{g} / \mathrm{mL}$ (Figura 7). (Insuasty, et al., 2015).<smiles>[Y17]C=C1CN([R])CC(=C[Al])C1=O</smiles><smiles>CSc1nc(N)cc(=O)[nH]1</smiles>

69
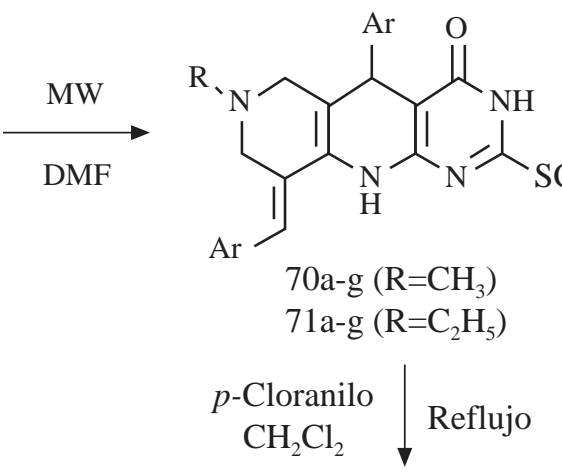

Figura 6. Síntesis de 6,7,8,9-tetrahidropirimido[4,5-b][1,6]naftiridin-4(3H,5H,10H)-onas 70a-g, 71a-g y sus formas oxidadas 72a-g, 73a-g. 
Ramírez, et al. (2015) reportaron la obtención de nuevas series de 8,9-dihidro-7H-pirimido[4,5- $b][1,4]$ diazepinas 84a-g y 85a-g ( Figura 24S, http://www.raccefyn.co/index. php/raccefyn/article/downloadSuppFile/309/1494), con alta regioselectividad a partir de la reacción entre triamino- o tetraaminopirimidinas $\mathbf{8 2}$ y $\mathbf{8 3}$ con compuestos carbonílicos $\alpha, \beta$-insaturados 81a-g. Doce de los compuestos sintetizados fueron seleccionados y probados por el US National Cancer Institute (NCI) para su actividad antitumoral contra 60 líneas celulares tumorales humanas diferentes. Los compuestos 85d y 85g mostraron importantes rangos de $\mathrm{GI}_{50}$ de 1.28$2.98 \mu \mathrm{M}$ y $0.35-2.78 \mu \mathrm{M}$ respectivamente bajo ensayos in vitro. Adicionalmente, las propiedades antifúngicas de 84ag y 85a-g fueron probadas contra los hongos clínicamente importantes Candida albicans y Cryptococcus neoformans. Aunque esos compuestos mostraron moderadas actividades contra C. albicans, los 2-amino derivados 85a-g y principalmente 85a y 85b, mostraron alta actividad contra $C$. neoformans con $\mathrm{MIC}_{50}=7.8-31.2 \mu \mathrm{g} / \mathrm{mL}, \mathrm{MIC}_{80}=15.6$ $31.2 \mu \mathrm{g} / \mathrm{mL}$ y $\mathrm{MIC}_{100}=15.6-62.5 \mu \mathrm{g} / \mathrm{mL}$.

Nuevas 2-alquilamino-6-aril-8,9-dihidropirimido[4,5-b][1,4] diazepin-4(7H)-onas 88a-o fueron preparadas regioselectivamente por la reacción entre 2-alquilamino-5,6diaminopirimidin-4(3H)-onas 86a-c y dimetilaminopropio fenonas (bases de Mannich) 87a-f (Figura 25S, http://www. raccefyn.co/index.php/raccefyn/article/downloadSuppFile/ 309/1495). (Insuasty, et al., 2014).

Tres series de nuevas 8,9-dihidro-7H-pirimido[5,4- $b$ ] [1,4]diazepinas, 90a-d, 91a-d, y 92a-d (Figura 26S, http:// www.raccefyn.co/index.php/raccefyn/article/download SuppFile/309/1496), fueron obtenidas eficientemente con buenos rendimientos usando metodologías de reacción simples. Estas pirimidodiazepinas fueron evaluadas contra 15 cepas de Mycobacterium spp. Se observó actividad moderada en la inhibición de 13 microorganismos para los compuestos 90a, 91a, 91c, y 91d. (Insuasty, et al., 2012b).
Una nueva serie de 6,6a,7,8-tetrahidro-5H-nafto[1,2-e] pirimido[4,5-b][1,4]diazepinas 94a-f y 95a-f fueron sintetizadas con buenos rendimientos a partir de la reacción entre E-2-ariliden-1-tetralonas 93 y la respectiva tri- o tetraaminopirimidinas 82 o 83 bajo radiación de microondas usando DMF como disolvente y cantidades catalíticas de $\mathrm{BF}_{3} \cdot \mathrm{OEt}_{2}$ (Figura 27S, http://www.raccefyn.co/index.php/ raccefyn/article/downloadSuppFile/309/1497). Seis de los compuestos obtenidos fueron selecciones y probados en 60 líneas celulares tumorales diferentes. En particular, los compuestos 95a, 95c y 95e presentaron remarcable actividad antitumoral contra cáncer de melanoma en la línea celular SK-MEL-5 con GI ${ }_{50}=0.50 \mu \mathrm{M}, \mathrm{GI}_{50}=0.609 \mu \mathrm{M}, \mathrm{GI}_{50}$ $=0.473 \mu \mathrm{M}$ respectivamente. (Insuasty, et al., 2010b).

Nuevas 11-amino-6-aril-6,7-dihidroindeno[1,2-e]pirimido $[4,5-b][1,4]$ diazepin-5(5aH)-onas 97a-f (Figura 28S, http://www.raccefyn.co/index.php/raccefyn/article/ downloadSuppFile/309/1498), fueron preparadas regioselectivamente por la reacción tricomponente de 4,5,6triaminopirimidina 82, 1,3-indandiona 96 y aldehídos aromáticos 12a-f. La aproximación bicomponente, usando 2,4,5,6-tetraaminopirimidina 83 y 2-arilidenindandionas 98a-f como reactivos, produjo 9,11-diamino-6-aril-6,7dihidroindeno[1,2-e]pirimido[4,5-b]-[1,4]diazepin5(5aH)-onas 99a-f con buenos rendimientos y los derivados regioisoméricos 8,10-diamino 100a-c con bajos rendimientos. Ambas aproximaciones fueron llevadas a cabo con radiación de microondas. (Insuasty, et al., 2008b).

Nuevas indeno[1,2-e]pirimido[4,5-b][1,4]diazepin-5,11dionas racémicas 101-127 fueron obtenidas regioselectivamente de la reacción entre 5,6-diamino-3,4-dihidropirimidin-4-onas 86 y 2-arilidenindandionas 98 como reactivos (Figura 29S, http://www.raccefyn.co/index. php/raccefyn/article/downloadSuppFile/309/1499). Estos compuestos fueron solicitados por el US National Cancer Institute (NCI) para evaluar su capacidad de inhibir

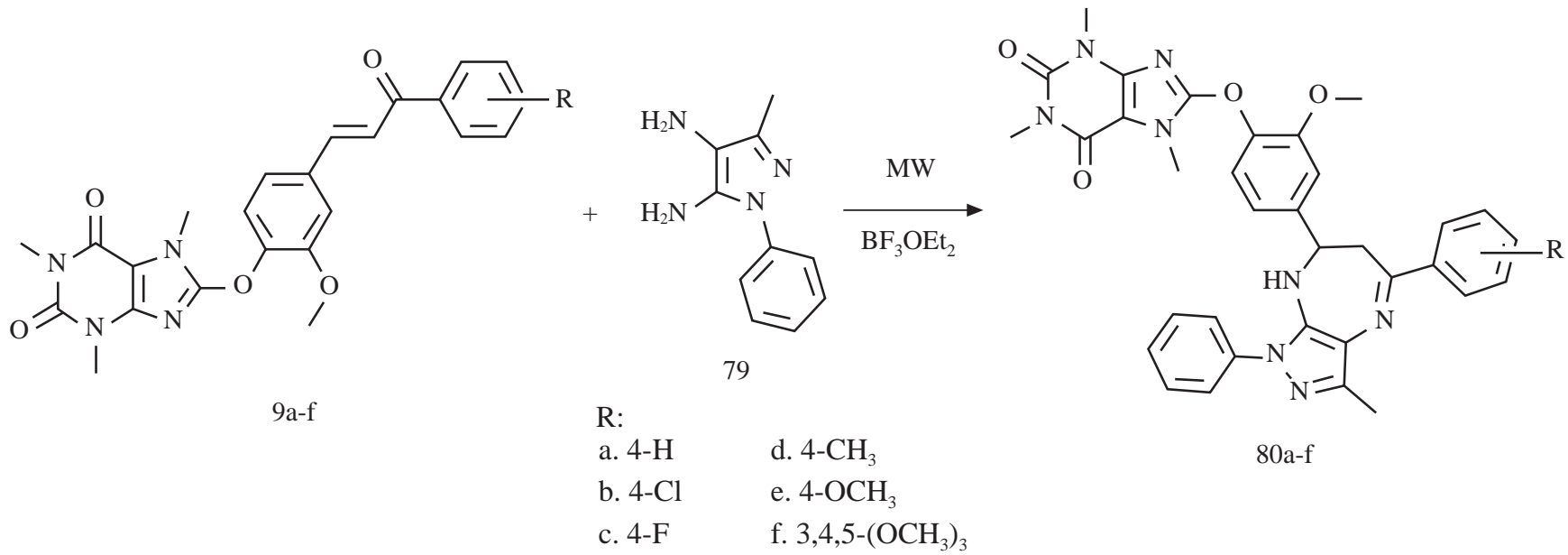

Figura 7. Síntesis de los derivados diazepínicos 80a-f a partir de la ciclocondensación regioselectiva de las chalconas 9a-f con el 4,5-diaminopirazol 79. 
aproximadamente 60 líneas celulares tumorales humanas diferentes, donde 103 y 104 presentaron importante actividad contra 57 y 48 líneas celulares tumorales, respectivamente, con los valores más importantes de $\mathrm{GI}_{50}$ en un rango desde 0.49 a $1.46 \mu \mathrm{M}$, en ensayos in vitro. (Insuasty, et al., 2008c).

Series de nuevas 4-amino-6-aril-8-(1,3-benzodioxol-5-il)8,9-dihidro-7 $H$-pirimido[4,5- $b][1,4]$ diazepinas 128a-f y 4-amino-8-aril-6-(1,3-benzodioxol-5-il)-8,9-dihidro-7Hpirimido $[4,5-b][1,4]$ diazepinas racémicas 129a-f fueron obtenidas regioselectivamente de la reacción entre 4,5,6triaminopirimidina 82 con 1 equivalente de metilendioxichalconas 47a-f y 51a-f, bajo radiación de microondas (Figura 30S, http://www.raccefyn.co/index.php/raccefyn/article/ downloadSuppFile/309/1500). La capacidad de inhibición de cerca de 60 líneas celulares tumorales humanas diferentes ha sido evaluada en el US National Cancer Institute (NCI), donde 128e, 129a y 129b presentaron remarcable actividad contra 47, 11 y 37 líneas celulares tumorales, respectivamente, con los valores más importantes de $\mathrm{GI}_{50}$ en un rango de 0.068 a $0.35 \mu \mathrm{M}$, en ensayos in vitro. (Insuasty, et al., 2008d).

\section{Conclusiones}

En este mini-review se reportan las contribuciones, más recientes, del autor y sus colaboradores en el área de la síntesis de chalconas y análogos mediante la condensación de Claisen-Schmidt y a partir de éstas la obtención de compuestos heterocíclicos de cinco (5) miembros (pirazolinas), de seis (6) miembros (piridinas) y de siete (7) miembros (diazepinas) mediante la reacción de Michael y posterior ciclocondensación. Algunos de los compuestos sintetizados presentan interesante actividad biológica, lo que conlleva a seguir investigando en el fascinante campo de la síntesis de nuevos compuestos con posible actividad biológica.

\section{Información suplementaria}

Figura 1S. Síntesis de flavonas $\mathbf{3}$ a partir de orto-hidroxichalconas 2. Ver figura suplementaria en: http://www.raccefyn.co/index.php/ raccefyn/article/downloadSuppFile/309/1471

Figura 2S. Adición directa (A) y conjugada (B) de un nucleófilo sobre un sistema carbonílico $\alpha, \beta$-insaturado. Ver figura suplementaria en: http://www.raccefyn.co/index.php/raccefyn/article/ downloadSuppFile/309/1472

Figura 3S. Formación de chalconas en medio ácido o básico. Ver figura suplementaria en: http://www.raccefyn.co/index.php/ raccefyn/article/downloadSuppFile/309/1473

Figura 4S. Obtención de las chalconas 13/14 derivadas de la 7-cloroquinolina. Ver figura suplementaria en: http:/www. raccefyn.co/index.php/raccefyn/article/downloadSuppFile/309/1474

Figura 5S. Nuevas (E)-1-aril-3-(3-aril-1-fenil-1H-pirazol-4il)-2-propen-1-onas $\mathbf{1 7 / 1 8}$ a partir de 3-aril-1-fenilpirazol-4carboxaldehídos 15/16. Ver figura suplementaria en: http://www. raccefyn.co/index.php/raccefyn/article/downloadSuppFile/309/1475

Figura 6S. Síntesis de derivados de tipo bis-chalconas 21 tratando las dicetonas 19/20 con aldehídos aromáticos 12a-c. Ver figura suplementaria en: http://www.raccefyn.co/index.php/raccefyn/ article/downloadSuppFile/309/1476

Figura 7S. Obtención de metilen-bis-chalconas 23a-e partiendo de acetofenonas sustituidas 8 y 5,5'-metilen-bis-salicilaldehído 22. Ver figura suplementaria en: http://www.raccefyn.co/index.php/ raccefyn/article/downloadSuppFile/309/1477

Figura 8S. Nuevos derivados hetaril- y alquilidenrodaninas 26a-d, 26e, 27a-d, 28a-d, 28e, y 29a-d preparados a partir de aldehídos heterocíclicos 24a-d o acetaldehído 24e. Ver figura suplementaria en: http://www.raccefyn.co/index.php/raccefyn/ article/downloadSuppFile/309/1478

Figura 9S. Síntesis asistida por microondas de nuevas (Z)-5arilidenrodaninas 30a-i. Ver figura suplementaria en: http://www. raccefyn.co/index.php/raccefyn/article/downloadSuppFile/309/1479
Figura 10S. Estructura y nomenclatura IUPAC de isómeros de las pirazolinas. Ver figura suplementaria en: http://www.raccefyn. co/index.php/raccefyn/article/downloadSuppFile/309/1480

Figura 11S. Síntesis y funcionalización de una nueva serie de $\mathrm{NH}$-pirazolinas 37 mediante la reacción entre [(7-cloroquinolin4-il)oxi]chalconas 36 con hidrato de hidrazina. Ver figura suplementaria en: http://www.raccefyn.co/index.php/raccefyn/article/ downloadSuppFile/309/1481

Figura 12S. Obtención de los compuestos 41a-f y posterior tratamiento con anhídrido acético o ácido fórmico. Ver figura suplementaria en: http://www.raccefyn.co/index.php/raccefyn/ article/downloadSuppFile/309/1482

Figura 13S. Nuevas series de $\mathrm{N}$-acetil- 44 y $\mathrm{N}$-formil-pirazolinas 45/46 sintetizadas por reacción entre [(7-cloroquinolin-4-il) amino]chalconas 13/14 con hidrato de hidrazina en ácido acético y ácido fórmico. Ver figura suplementaria en: http://www.raccefyn. co/index.php/raccefyn/article/downloadSuppFile/309/1483

Figura 14S. Síntesis de nuevas 2-pirazolinas 1,3,5-trisustituidas conteniendo el residuo de benzodioxol. Ver figura suplementaria en: http://www.raccefyn.co/index.php/raccefyn/article/download SuppFile/309/1484

Figura 15S. Síntesis asistida por microondas de los 3-aril-4-(3aril-4,5-dihidro-1H-pirazol-5-il)-1-fenil- $1 H$-pirazoles racémicos 55 o sus $N$-acetil derivados 56/57. Ver figura suplementaria en: http://www.raccefyn.co/index.php/raccefyn/article/download SuppFile/309/1485

Figura 16S. Nuevos derivados bis-3,5-difenilpirazolínicos 58a-f sintetizados a partir de la reacción entre bis-chalconas enlazadas por un grupo alquilo 21a-f e hidrazina en ácido acético. Ver figura suplementaria en: http://www.raccefyn.co/index.php/raccefyn/ article/downloadSuppFile/309/1486 
Figura 17S. Síntesis de nuevas series de 4,4'-metilenbis [2-(3-aril4,5-dihidro-1H-pirazol-5-il) fenoles] 59 y metilenbis-2-(3-aril4,5-dihidro-1H-pirazol-5-il)-4,1-fenilen diacetatos $\mathbf{6 0}$. Ver figura suplementaria en: http://www.raccefyn.co/index.php/raccefyn/article/ downloadSuppFile/309/1487

Figura 18S. Formación de derivados dihidropiridínicos fusionados a partir de chalconas. Ver figura suplementaria en: http://www. raccefyn.co/index.php/raccefyn/article/downloadSuppFile/309/1488

Figura 19S. Condensación de aminopirazoles $N H$-sustituidos 61 y NH-no sustituidos 62 con chalconas 1 y posterior aromatización de los productos con NBS. Ver figura suplementaria en: http://www. raccefyn.co/index.php/raccefyn/article/downloadSuppFile/309/1489

Figura 20S. Estructuras de los isómeros de diazepinas. Ver figura suplementaria en: http://www.raccefyn.co/index.php/raccefyn/ article/downloadSuppFile/309/1490

Figura 21S. Formación de benzodiazepinas por medio de la reacción entre orto-fenilendiamina y chalconas. Ver figura suplementaria en: http://www.raccefyn.co/index.php/raccefyn/article/ downloadSuppFile/309/1491

Figura 22S. Formación de regioisómeros por la reacción entre orto-diaminas con dos grupos amino no equivalentes y chalconas. Ver figura suplementaria en: http://www.raccefyn.co/index.php/ raccefyn/article/downloadSuppFile/309/1492

Figura 23S. Formación de dihidrodiazepinas fusionadas a partir de chalconas. Ver figura suplementaria en: http://www.raccefyn.co/ index.php/raccefyn/article/downloadSuppFile/309/1493

Figura 24S. Obtención de nuevas series de 8,9-dihidro-7Hpirimido[4,5-b][1,4]diazepinas 84a-g y 85a-g. Ver figura suplementaria en: http://www.raccefyn.co/index.php/raccefyn/article/ downloadSuppFile/309/1494
Figura 25S. Síntesis de nuevas 2-alquilamino-6-aril-8,9dihidropirimido[4,5-b][1,4]diazepin-4(7H)-onas 88a-o usando dimetilaminopropiofenonas (bases de Mannich) 87a-f. Ver figura suplementaria en: http://www.raccefyn.co/index.php/raccefyn/ article/downloadSuppFile/309/1495

Figura 26S. Obtención de tres series de nuevas 8,9-dihidro-7Hpirimido[5,4- $b][1,4]$ diazepinas, 90a-d, 91a-d, y 92a-d. Ver figura suplementaria en: http://www.raccefyn.co/index.php/raccefyn/article/ downloadSuppFile/309/1496

Figura 27S. Síntesis de las 6,6a,7,8-tetrahidro-5H-nafto[1,2-e] pirimido[4,5-b][1,4]diazepinas 94a-f y 95a-f usando radiación de microondas y cantidades catalíticas de $\mathrm{BF}_{3}$. OEt2. Ver figura suplementaria en: http://www.raccefyn.co/index.php/raccefyn/article/ downloadSuppFile/309/1497

Figura 28S. Formación de nuevas diazepinas fusionadas derivadas de la indandiona. Ver figura suplementaria en: http://www.raccefyn. co/index.php/raccefyn/article/downloadSuppFile/309/1498

Figura 29S. Nuevas indeno[1,2-e]pirimido[4,5-b][1,4]diazepin5,11-dionas racémicas 101-127 obtenidas regioselectivamente a partir de 2-arilidenindandionas 98. Ver figura suplementaria en: http://www.raccefyn.co/index.php/raccefyn/article/download SuppFile/ 309/1499

Figura 30S. Síntesis de nuevas 4-amino-6-aril-8-(1,3-benzodioxol5-il)-8,9-dihidro-7H-pirimido[4,5-b][1,4]diazepinas 128a-f y 4amino-8-aril-6-(1,3-benzodioxol-5-il)-8,9-dihidro-7 H-pirimido [4,5-b][1,4]diazepinas racémicas 129a-f. Ver figura suplementaria en: http://www.raccefyn.co/index.php/raccefyn/article/download SuppFile/309/1500

\section{Agradecimientos}

B.I.O. agradece a todos los integrantes del Grupo de Investigación de Compuestos Heterocíclicos (GICH) de la Universidad del Valle. B.I.O. igualmente agradece a sus 59 estudiantes, quienes han realizado o están realizando sus tesis de doctorado (12), sus trabajos de investigación de maestría (12) o sus trabajos de grado (35), en especial a Jonathan Ramírez Prada y Viviana Cuartas Granada por su colaboración. A la Dra. Susana Zacchino, al Dr. Iván Darío Vélez, a la Dra Sara Robledo, a los Drs. Manuel Nogueras y Justo Cobo de la Universidad de Jaén, España y al Instituto Nacional de Cancerología (NCI) de los Estados Unidos.

Adicionalmente, agradece a COLCIENCIAS, Banco de la República y Universidad del Valle por el soporte financiero a su investigación.

Gracias a la colaboración del National Cancer Institute (NCI) de los Estados Unidos, a la Universidad Nacional de Rosario en Argentina y a la Universidad de Antioquia en Colombia se han estudiado la actividad antitumoral, antifúngica y antiparasitaria de algunos de los nuevos compuestos sintetizados.

\section{Conflicto de intereses}

El autor declara no tener conflicto de intereses.

\section{Bibliografía}

Anand, P., Singh, B. (2014). Pyrrolo-isoxazole: A Key Molecule with Diverse Biological Actions. Mini-Reviews in Medicinal Chemistry, 14 (7): 623-627.

Awad, W., El-Neweihy, M., Selim, F. (1960). Studies on 3-Acylcatechols. II. A New Synthesis of 8-Hydroxyflavone. The Journal of Organic Chemistry, 25 (8): 1333-1336.

Insuasty, B., Ramírez, J., Becerra, D., Echeverry, C., Quiroga, J., Abonia, R., Robledo, S. M., Vélez, I. D., Upegui, Y., Muñoz, J. A., Ospina, V., Nogueras, M., Cobo, J. (2015). An efficient synthesis of new caffeine-based chalcones, pyrazolines and pyrazolo[3,4-b][1,4]diazepines as potential antimalarial, antitrypanosomal and antileishmanial agents. European Journal of Medicinal Chemistry, 93: 401-413.

Insuasty, B., Orozco, F., Quiroga, J., Abonia, R., Nogueras, M., Cobo, J. (2014). Synthesis and Structure Elucidation of New Regioisomeric 2-Alkylamino-6-aryl-8,9-dihydropyrimido [4,5-b][1,4]diazepin-4(7H)-ones. Journal of Heterocyclic Chemistry, 51 (1): 196-202. 
Insuasty, B., Montoya, A., Becerra, D., Quiroga, J., Abonia, R., Robledo, S., Vélez, I. D., Upegui, Y., Nogueras, M., Cobo, J. (2013a). Synthesis of novel analogs of 2-pyrazoline obtained from [(7-chloroquinolin-4-yl)amino]chalcones and hydrazine as potential antitumor and antimalarial agents. European Journal of Medicinal Chemistry, 67: 252-262.

Insuasty, A., Ramírez, J., Raimondi, M., Echeverry, C., Quiroga, J., Abonia, R., Nogueras, M., Cobo, J., Rodríguez, M. V., Zacchino, S. A., Insuasty, B. (2013b). Synthesis, Antifungal and Antitumor Activity of Novel (Z)-5-Hetarylmethylidene1,3-thiazol-4-ones and (Z)-5-Ethylidene-1,3-thiazol-4-ones. Molecules, 18 (5): 5482-5497.

Insuasty, B., Becerra, D., Quiroga, J., Abonia, R., Nogueras, M., Cobo, J. (2013c). Microwave-assisted synthesis of pyrimido[4,5-b][1,6]naphthyridin-4(3H)-ones with potential antitumor activity. European Journal of Medicinal Chemistry, 60: 1-9.

Insuasty, B., Chamizo, L., Muñoz, J., Tigreros, A., Quiroga, J., Abonia, R., Nogueras, M., Cobo, J. (2012a). Synthesis of 1-Substituted 3-Aryl-5-aryl(hetaryl)-2-pyrazolines and Study of Their Antitumor Activity. Archiv der Pharmazie, 345 (4): 275-286.

Insuasty, B., García, A., Bueno, J., Quiroga, J., Abonia, R., Ortiz, A. (2012b). Antimycobacterial Activity of Pyrimido[4,5-b] diazepine Derivatives. Archiv der Pharmazie, 345 (9): 739-744.

Insuasty, B., García, A., Quiroga, J., Abonia, R., Ortiz, A., Nogueras, M., Cobo, J. (2011). Efficient microwaveassisted synthesis and antitumor activity of novel 4,4'-methylenebis[2-(3-aryl-4,5-dihydro-1H-pyrazol-5-yl) phenols]. European Journal of Medicinal Chemistry, 46 (6): 2436-2440.

Insuasty, B., Tigreros, A., Orozco, F., Quiroga, J., Abonia, R., Nogueras, M., Sanchez, A., Cobo, J. (2010a). Synthesis of novel pyrazolic analogues of chalcones and their 3-aryl-4-(3-aryl-4,5-dihydro- $1 H$-pyrazol-5-yl)-1-phenyl$1 H$-pyrazole derivatives as potential antitumor agents. Bioorganic \& Medicinal Chemistry, 18 (14): 4965-4974.

Insuasty, B., García, A., Quiroga, J., Abonia, R., Nogueras, M., Cobo, J. (2010b). Synthesis of novel 6,6a,7,8-tetrahydro$5 H$-naphtho[1,2-e]pyrimido[4,5-b][1,4]diazepines under microwave irradiation as potential anti-tumor agents. European Journal of Medicinal Chemistry, 45 (7): 28412846.

Insuasty, B., Martinez, H., Quiroga, J., Abonia, R., Nogueras, M., Cobo, J. (2008a). Synthesis of new bis-3,5diphenylpyrazolines derivatives linked with alkyl chains. Journal of Heterocyclic Chemistry, 45 (5): 1521-1524.

Insuasty, B., Orozco, F., García, A., Quiroga, J., Abonia, R., Nogueras, M., Cobo, J. (2008b). Microwave-assisted synthesis of new regioisomeric 6,7-dihydroindeno[1,2-e] pyrimido[4,5- $b][1,4]$ diazepin-5(5aH)-ones. Journal of Heterocyclic Chemistry, 45 (6): 1659-1663.

Insuasty, B., Orozco, F., Lizarazo, C., Quiroga, J., Abonia, R., Hursthouse, M., Nogueras, M., Cobo, J. (2008c). Synthesis of new indeno[1,2-e]pyrimido[4,5-b][1,4] diazepine-5,11-diones as potential antitumor agents. Bioorganic \& Medicinal Chemistry, 16 (18): 8492-8500.
Insuasty, B., Orozco, F., Quiroga, J., Abonia, R., Nogueras, M., Cobo, J. (2008d). Microwave induced synthesis of novel 8,9-dihydro-7H-pyrimido[4,5-b][1,4]diazepines as potential antitumor agents. European Journal of Medicinal Chemistry, 43 (9): 1955-1962.

Insuasty, B., Abonia, R., Quiroga, J. (1992). The reaction of ketones with ortho-diamines. I. The reaction of aromatic $\alpha, \beta$-unsaturated ketones with 4,5-dimethyl-1,2phenylenediamine. An. Quim., 88: 718-721.

Kostanecki, S., Tambor, J. (1899). Ueber die sechs isomeren Monooxybenzalacetophenone (Monooxychalkone). Berichte der deutschen chemischen Gesellschaft, 32 (2): 1921-1926.

Mohamed, R., Abdelrahman, S., Ahmad, M. (2012). Recent advances in the therapeutic applications of pyrazolines. Expert Opinion on Therapeutic Patents, 22 (3): 253-291.

Montoya, A., Quiroga, J., Abonia, R., Nogueras, M., Cobo, J., Insuasty, B. (2014). Synthesis and in Vitro Antitumor Activity of a Novel Series of 2-Pyrazoline Derivatives Bearing the 4-Aryloxy-7-chloroquinoline Fragment. Molecules, 19 (11): 18656-18675.

Parvesh, S., Amit, A., Vipan, K. (2014). Recent developments in biological activities of chalcones: A mini review. European Journal of Medicinal Chemistry, 85: 758-777.

Quiroga, J., Insuasty, B., Rincón, R., Larrahondo, M., Hanold, N., Meier, H. (1994). The formation of pyrazolo[1,5-a] pyrimidines by the reaction of 3-(4-chlorophenyl) pyrazol-5-amine with chalcones. Journal of Heterocyclic Chemistry, 31 (6): 1333-1335.

Ramajayam, R., Girdhar, R., Yadav, M. (2007). Current Scenario of 1,4-Diazepines as Potent Biomolecules-A Mini Review. Mini-Reviews in Medicinal Chemistry, 7 (8): 793-812.

Ramírez, J., Svetaz, L., Quiroga, J., Abonia, R., Raimondi, M., Zacchino, S., Insuasty, B. (2015). Synthesis of novel thiazole-based 8,9-dihydro-7H-pyrimido[4,5-b][1,4] diazepines as potential antitumor and antifungal agents. European Journal of Medicinal Chemistry, 92: 866-875.

Ramírez, J., Rodríguez, M. V., Quiroga, J., Abonia, R., Sortino, M., Zacchino, S. A., Insuasty, B. (2014). Efficient Synthesis of Novel 3-Aryl-5-(4-chloro-2-morpholinothiazol-5-yl)4,5-dihydro- $1 H$-pyrazoles and Their Antifungal Activity Alone and in Combination with Commercial Antifungal Agents. Archiv der Pharmazie, 347 (8): 566-575.

Sortino, M., Delgado, P., Juárez, S., Quiroga, J., Abonia, R., Insuasty, B., Nogueras, M., Rodero, L., Garibottoe, F. M., Enrize, R. D., Zacchino, S. (2007). Synthesis and antifungal activity of (Z)-5-arylidenerhodanines. Bioorganic \& Medicinal Chemistry, 15 (1): 484-494.

Vianna-Rodrigues, S., Martins-Viana, L., Quiroga, J., Insuasty, B., Abonia, R., Baumann, W. (1994). Chromatographic and spectroscopic properties of regioisomers of some $1 \mathrm{H}$ 1,5-benzodiazepines. Journal of Heterocyclic Chemistry, 31 (4): 813-817.

Wenglowsky, S. (2013). Pyrazolo[3,4-b]pyridine kinase inhibitors: a patent review (2008 - present). Expert Opinion on Therapeutic Patents, 23 (3): 281-298. 